\title{
Overcoming Back Electron Transfer Facilitates the Implementation of Electron Donor- Acceptor Complexes in Catalysis
}

Edward J. McClain, Timothy M. Monos, Mayuko Mori, Joel W. Beatty, Corey R. J. Stephenson*

Willard Henry Dow Laboratory, Department of Chemistry, University of Michigan, 930 North University Avenue, Ann Arbor, Michigan 48109, United States.

*Correspondence to: crjsteph@umich.edu

\begin{abstract}
Electron donor-acceptor (EDA) complexes can controllably generate radicals under mild conditions through selective photoexcitation events. However, unproductive reactivity from fast deactivation of the photoexcited complexes through back electron transfer has slowed the development of EDA complexes in synthetic methodology. Here, we disclose the study of EDA complexes derived from 2-methoxynaphthalene donor and acylated ethyl isonicotinate $N$-oxide acceptor that undergo a fast $\mathrm{N}-\mathrm{O}$ bond fragmentation event upon photoexcitation. This reaction design not only overcomes the limitations of back electron transfer but also enables the regeneration of the donor species, representing a rare example EDA photochemistry in a catalytic regime. The synthetic utility is demonstrated through visible light-driven radical trifluoromethylation and Minisci alkylation reactions. The scalability of the EDA complex promoted reaction evidenced by the successful multigram-scale trifluoromethylation of methyl N-Boc pyrrole-2-carboxylate in a continuous flow manifold.
\end{abstract}

Main Text: Electron donor-acceptor (EDA) complexes are ground state aggregates that are characterized by the formation of a red-shifted transition referred to as a charge transfer band (1). Photoexcitation of this transition induces an electron transfer event that produces a radical ion pair. These ion pairs typically return to the ground state via a rapid back electron transfer, leaving little opportunity for either radical ion component to engage in productive reactivity (2). To overcome these challenges, EDA complexes employed in synthesis are designed to incorporate a labile bond that fragments upon photoexcitation at a rate competitive with back electron transfer (3). The rapid bond scission event decomposes one component of the contact ion pair, facilitating the otherwise improbable cage escape and thus enabling potential downstream radical reactivity. Pioneering studies by Kochi and co-workers exemplify this reactivity paradigm, as EDA complexes derived from pyridinium salts were demonstrated

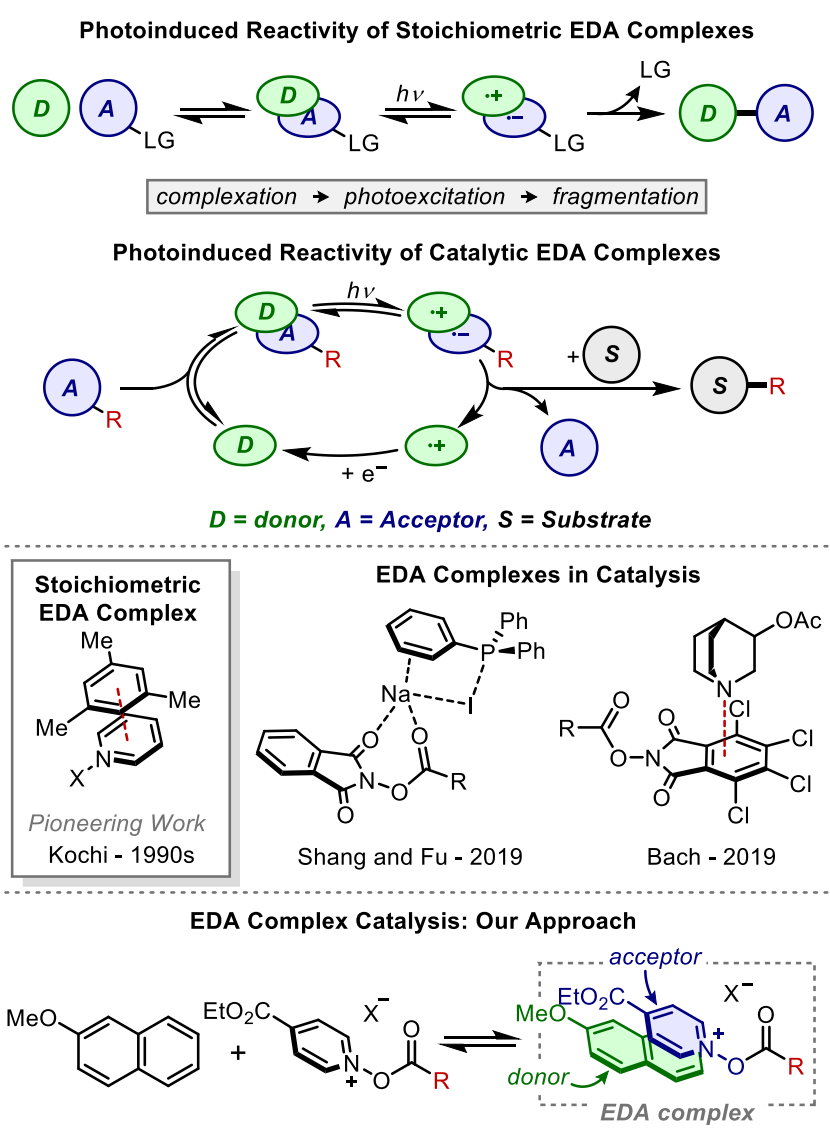

Figure 1. Overview of EDA complex catalysis. 
to undergo rapid fragmentation of the $\mathrm{N}-\mathrm{X}$ bond upon photoexcitation, facilitating the nitration and fluorination of the aromatic donor molecules (4).

Despite the long-standing interest in the stoichiometric photoinduced reactivity of charge transfer complexes, reaction manifolds that implement EDA complexes as catalytic intermediates remain severely under-explored (3a). The development of EDA complex catalysis represents an important advancement in this field, as it will expand the scope of reactivity by decoupling the complexation and photogeneration of radicals from substrate choice/functionalization (Figure 1). In 2013, Melchiorre and co-workers demonstrated that organocatalysis paired with photo-induced EDA complex reactivity could achieve the asymmetric $\alpha$-alkylation of aldehydes (5). This reactivity was enabled by the formation of a highly polarized, electron rich enamine as a catalytic intermediate that could associate with electron deficient nitrobenzyl bromides and $\alpha$-bromoacetophenones (Figure 1). Subsequent efforts have further demonstrated the utility of coupling organocatalysis with EDA complex photochemistry (6). In 2019, Shang and Fu reported a strategy for EDA complex catalysis that utilized the combination of triphenylphosphine with sodium iodide as a catalyst species (7). Photoexcitaiton of the chargetransfer complexes formed from the triphenylphosphine/sodium iodide catalyst species with $\mathrm{N}$ hydroxyphthalimide redox auxiliaries, Katritzky's N-alkyl pyridinium salts, or Togni's reagent promoted alkylation or trifluoromethylation reactions, respectively (Figure 1). The same year, Bach and Bosque showed that 3-acetoxyquinuclidine could function as a catalyst in the presence of tetrachlorophthalamide redox active esters, delivering amino- and hydro-decarboxylation as well as cyclization and dimerization reactions upon irradiation of the EDA complex (Figure 1) (8).

In 2015, our group reported the application of trifluoroacetic anhydride and pyridine $N$-oxide as a cheap, readily available, easily handled reagent combination for the radical trifluoromethylation of electron-rich (hetero)arenes (9). During this study, it was discovered that a background trifluoromethylation reaction was occurring in the absence of a photocatalyst. UV-vis studies revealed that the reaction was promoted by a stoichiometric EDA complex between the aromatic substrate (donor) and $N$-(trifluoroacetoxy)pyridinium (acceptor). We saw this as an opportunity to develop a generalized catalytic reaction manifold predicated on the formation and photoexcitation of an EDA complex as a catalytic intermediate. The application of an exogenous donor catalyst allows for the formation of a highly modular EDA complex, imparting the ability to tune the charge transfer band energy for selective photoexcitation events. Additionally, this reaction design delivers the radical intermediate through an EDA complex derived from a $\pi$-stacking interaction $(2 b)$, providing
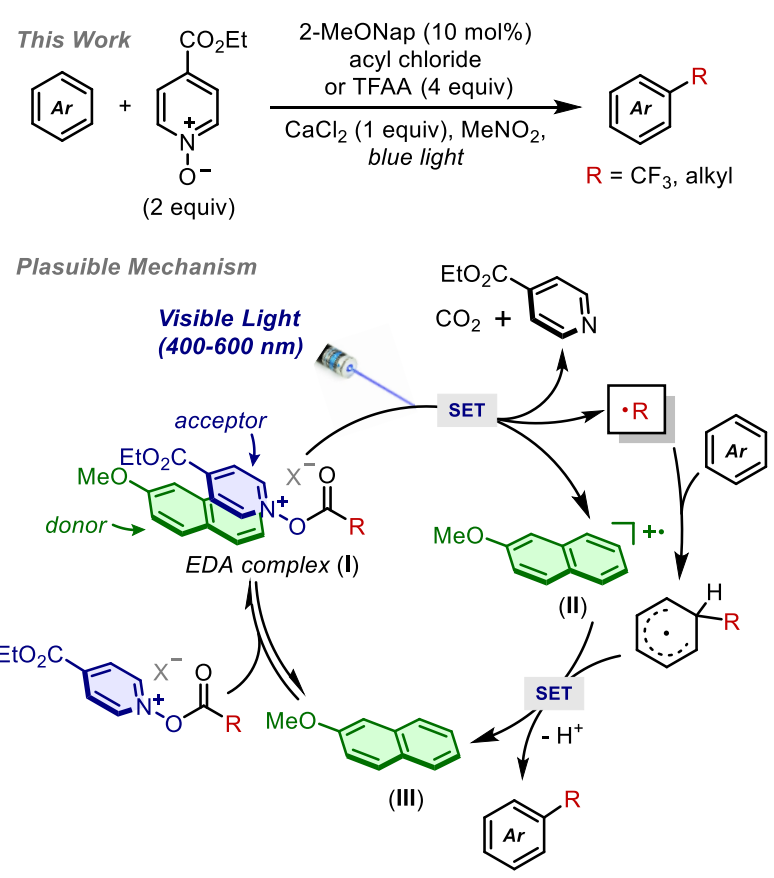

Figure 2. Plausible mechanism for EDA complex catalysis. 
complementary reactivity to perfluoroalkylation methods that employ nitrogenous bases for the formation of an EDA complex (10), while allowing for more diverse, less polarized radical fragments to be accessed.

Upon realization of a $\pi$-stacking interaction that delivers the desired EDA complex (I), we envisioned that a catalytic manifold may be accessible as the reversible $\pi$-stacking interaction allows for facile entry into the catalytic cycle. Photoexcitation of the EDA complex (I) induces an electron transfer event that produces 2methoxynaphthalene radical cation (II) and liberates an equivalent of the reactive radical intermediate. In the presence of an arene, the radical can be efficiently trapped. Subsequent oxidation and deprotonation of the radical addition adduct produces the desired $\mathrm{C}-\mathrm{H}$ trifluoromethylated product and regenerates 2-methoxynaphthalene (III) to turn over the catalytic cycle (Figure 2).
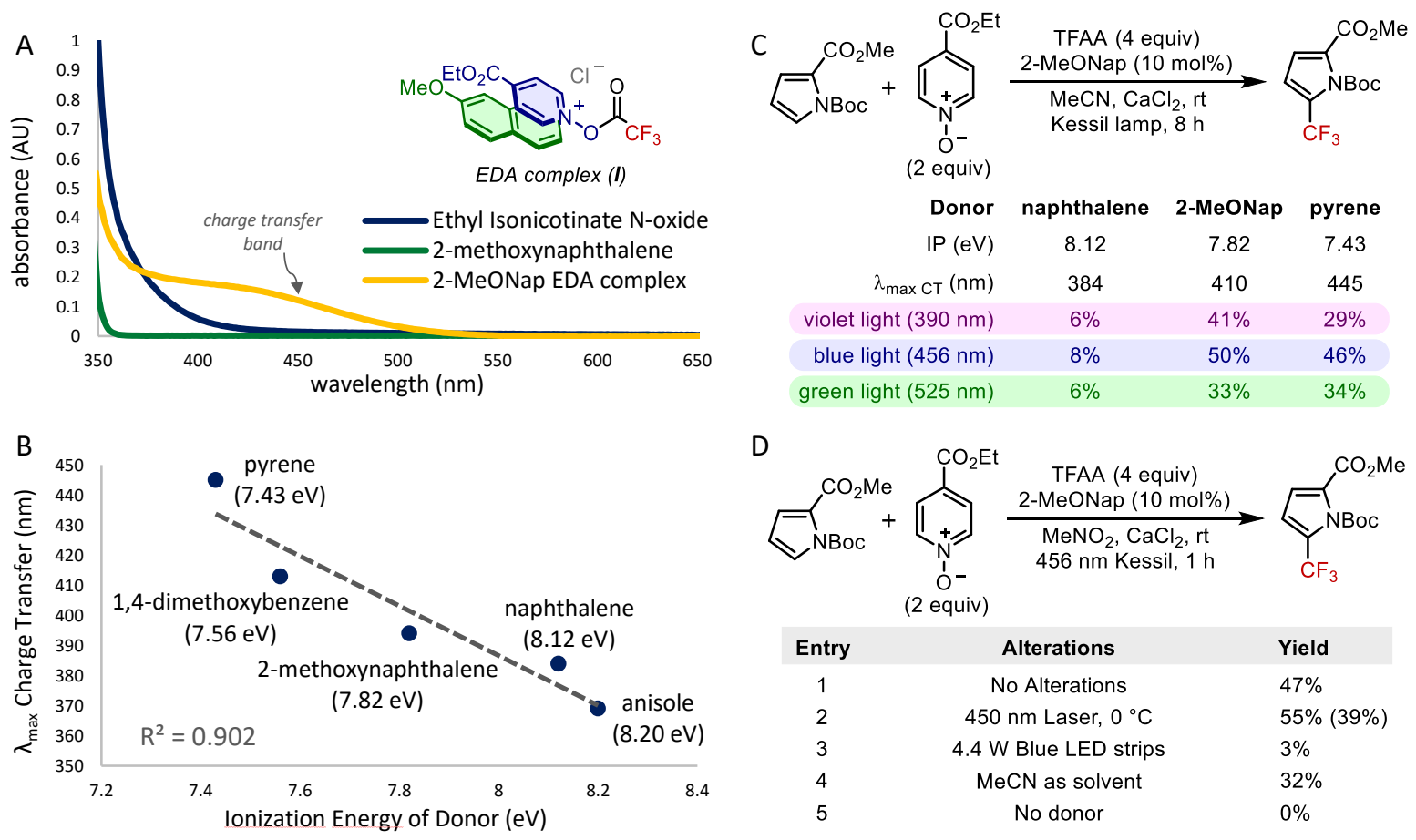

Figure 3. A) UV-vis spectra for EDA complex of 2-methoxynaphthalene with ethyl $N$-(trifluoroacetoxy)pyridinium-4-carboxylate. B) Mulliken correlation of arene donors with ethyl $N$-(trifluoroacetoxy)pyridinium-4-carboxylate in acetonitrile. C) Effect of irradiation wavelength on reactivity of EDA complexes. ${ }^{19} \mathrm{~F}$ NMR yields with $\mathrm{PhCF}_{3}$ as internal standard. D) Optimization of reaction conditions. ${ }^{19} \mathrm{~F}$ NMR yields with $\mathrm{PhCF}_{3}$ as internal standard (Isolated yield).

Initial investigations into the development of a catalytic manifold focused on the optimization of the chargetransfer complex that will serve as a catalytic intermediate. Preliminary studies revealed that donors with extended $\pi$-systems (i.e. naphthalene) provided more absorbing EDA complexes, while the incorporation of an electron donating substituent increased observed reactivity of the EDA complex. EDA complexes are characterized by Mulliken's theory of charge transfer, a postulate that relates the energy of the charge transfer band ( $\left.\mathrm{E}_{\mathrm{CT}}\right)$ for the complex to the difference between the donor ionization energy (IE) and acceptor electron affinity (EA) (11). Studies of the acceptor revealed ethyl isonicotinate N-oxide exhibited the formation of an EDA complex with anisole that provided a charge transfer band with a maximum absorbance at $369 \mathrm{~nm}$. Altering the donor arene in acetonitrile led to the formation of new, red-shifted charge transfer bands. As is predicted by Mulliken theory, $\mathrm{E}_{\mathrm{CT}}$ decreased as the IE of the donor decreased (Figure 3B). Importantly, more red-shifted charge transfer bands allow 
for highly selective excitation of the desired transition, a facet controllable through the choice of donor catalyst and light source (Figure 3). For instance, in the presence of 2-methoxynaphthalene or pyrene as donor catalysts, optimal yields were obtained when applying blue light irradiation (456 nm). However, irradiation with either violet $(390 \mathrm{~nm})$ or green $(525 \mathrm{~nm})$ light negatively impacted reactivity (Figure 3C).

Further studies of the EDA complex demonstrated that the absorbance of the charge transfer band increased with the polarity of the solvent and concentration, as the complex showed peak absorbance properties in nitromethane at or above $0.6 \mathrm{M}$. Interestingly, it was found that the addition of an inorganic salt, such as calcium chloride, had a modest impact on the absorbance of the complex, as the addition of 1 equivalent of calcium chloride in nitromethane delivered the strongest absorbance for the CT band. It is believed that the Lewis acidic metal plays a dual role in increasing the absorptivity of the EDA complex, by both modifying the acceptor through coordination and by acting as an electrolyte, thereby increasing the ionic strength of the solution. Analysis of the complex using Job's method (12) demonstrated that the most absorbing species is a 4:1 complex of 2methoxynaphthalene donor to ethyl $\mathrm{N}$ (trifluoroacetoxy)pyridinium-4-carboxylate acceptor in nitromethane (Figure 4). Precedence for EDA complexes that deviate from a 1:1 stoichiometry is found in Kochi's work, in which it was demonstrated that pyrene and pyridine $\mathrm{N}$-oxide in the presence of Lewis acid boron

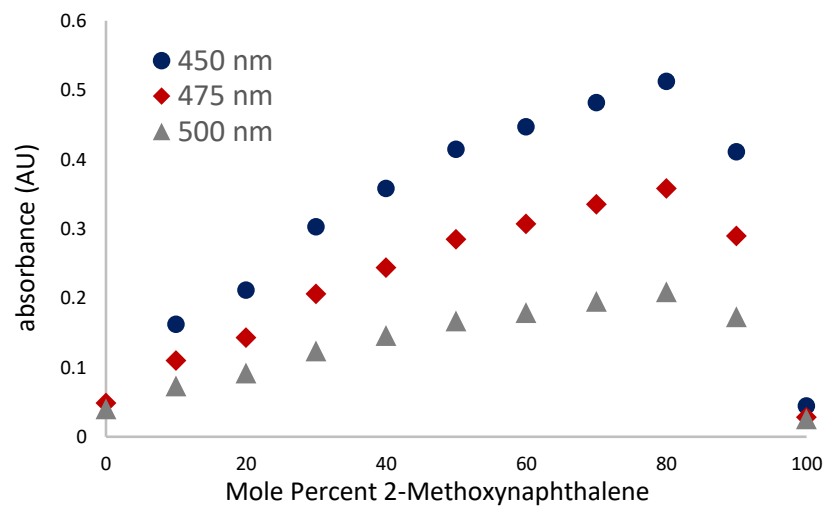

Figure 4. Job plot of 2-methoxynaphthalene (donor) with ethyl $\quad N$-(trifluoroacetoxy)pyridinium-4-carboxylate (acceptor). (•) Absorbance at $450 \mathrm{~nm}$. ( $\bullet$ Absorbance at $475 \mathrm{~nm}$. (A) Absorbance at $500 \mathrm{~nm}$. trifluoride form 2:1:1 complexes (13). It should be noted that while our observations support a 4:1 donor:acceptor stoichiometry, many states could exist in solution at any given time, and it is unclear which of these is the most reactive form of the EDA complex under the reaction conditions. 
Applying knowledge gained from the aforementioned studies of the EDA complex, investigation of the trifluoromethylation reaction revealed that optimal yields (55\% 3a) were obtained in the presence of $10 \mathrm{~mol} \% 2$ methoxynaphthalene donor, calcium chloride (1 equiv), ethyl isonicotinate $\mathrm{N}$-oxide ( 2 equiv) and an excess of trifluoroacetic anhydride (4 equiv) under irradiation from a $450 \mathrm{~nm}$ laser diode (Figure 3D). Notably, this result is on par with the yield obtained when carrying out the radical trifluoromethylation under $\mathrm{Ru}(\mathrm{bpy})_{3} \mathrm{Cl}_{2}$ catalyzed
Scheme 1. Reaction inhibition by ethyl isonicotinate

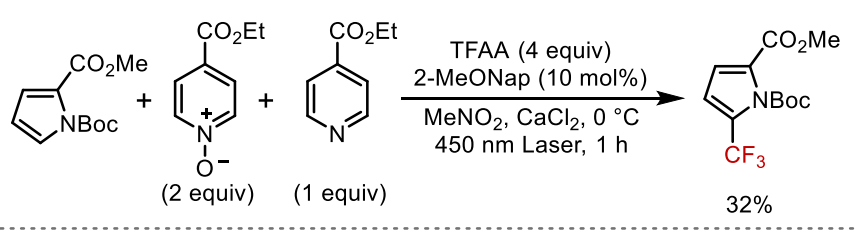

Unproductive EDA complex

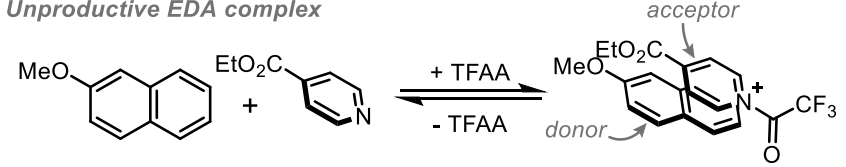
isonicotinate $\mathrm{N}$-oxide/TFAA did not lead to increased yields of the $\mathrm{C}-\mathrm{H}$ trifluoromethylation products, but instead resulted in the production of the trifluoroacetylated side product, which arises from nucleophilic trapping of the electrophilic ethyl $N$-(trifluoroacetoxy)pyridinium-4-carboxylate intermediate. A second addition of 2 equiv. of ethyl isonicotinate $N$-oxide and 4 equiv. TFAA reagent mixture in nitromethane, followed by irradiation for an additional hour, did not improve the reaction yield. We hypothesized that under reaction conditions the production of ethyl isonicotinate caused the reaction to stall. To test this hypothesis, we carried out the reaction in the presence of 1 equivalent of ethyl isonicotinate. Indeed, the addition of ethyl isonicotinate has a deleterious effect on the reaction outcome (Scheme 1). And further investigations revealed that an unproductive EDA complex forms with ethyl $N$-(trifluoroacetyl)pyridinium-4-carboxylate that is produced under reaction conditions following
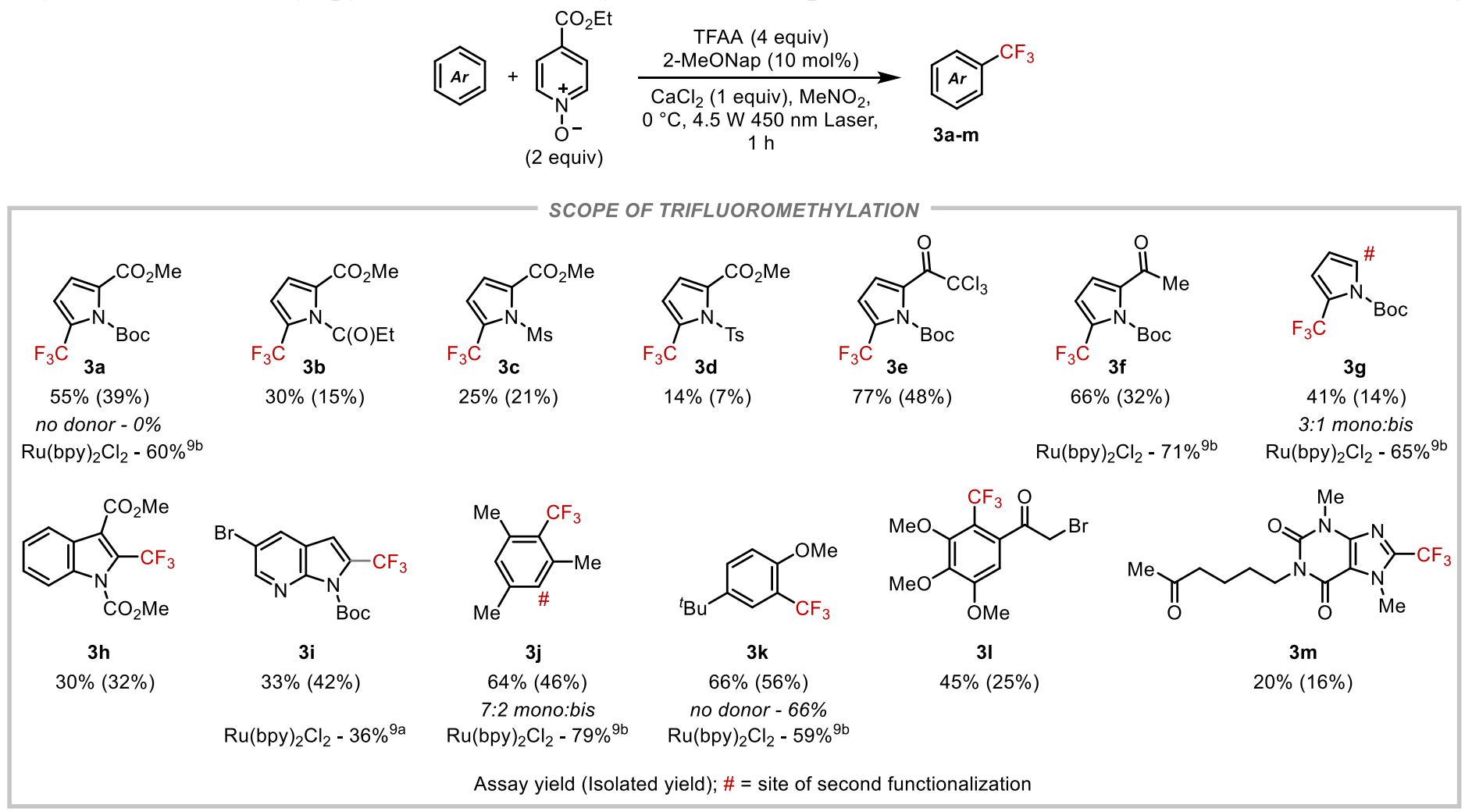

Figure 5. Scope of EDA complex catalyzed radical trifluoromethylation. Standard conditions: Arene $(0.2 \mathrm{mmol}, 1 \mathrm{equiv})$, EINO (2 equiv), TFAA (4 equiv), 2-methoxynaphthalene (10 mol\%), calcium chloride (1 equiv), nitromethane $(0.25 \mathrm{~mL}, 0.8 \mathrm{M}), 0{ }^{\circ} \mathrm{C}, 450$ $\mathrm{nm}$ blue laser, 1 hour. Assay yields determined by ${ }^{19} \mathrm{~F} \mathrm{NMR}$ with $\mathrm{PhCF}_{3}$ as internal standard (Isolated yield). $\mathrm{Ru}(\mathrm{bpy}) \mathrm{Cl}_{2} \mathrm{catalyzed}$ yields from reference 9 . 
decarboxylation. It is hypothesized that this complex sequesters donor catalyst away from the designed EDA complex, and absorbs photons that would otherwise be absorbed by the key catalytic intermediate.

Exploration of the scope of the trifluoromethylation reaction demonstrated that $N$-protecting groups had an influence on the reactivity, as $N$-Boc protected methyl pyrrole-2-carboxylate gave the highest yield (55\%, 3a). While $N$-mesyl (3c) and $N$-tosyl (3d) protecting groups had a significant deleterious impact on the reaction. $N$ Boc pyrrole (3g) proceeded in $41 \%$ yield with a 3 to 1 mixture of mono to bis trifluoromethylation. $N$-Boc 2 acetylpyrrole (3f) proceeded in good yield providing $66 \%$ of the trifluoromethylated product. $N$-Boc 2(trichloro)acetylpyrrole (3e) and $\alpha$-bromoacetophenone (3I) also provided the trifluoromethylated products in good yields, notably retaining the $\alpha$-halogenation. Substituted benzene derivatives were effective substrates for the trifluoromethylation reaction, providing the trifluoromethylated aromatics in good yields $(\mathbf{3 j} \mathbf{j}-\mathbf{3 l})$. Finally, moderately complex indole $(\mathbf{3 h})$ and $N$-Boc-5-bromo-7-azaindole (3i) provided the trifluoromethylated products in modest yield as single-isomers.

Given the low costs of reagents and access to process friendly materials the reported EDA complex catalyzed trifluoromethylation may be an attractive reaction for process chemists, as such, we sought to explore the scalability of the reaction. Initial scale-up efforts failed, as in the presence of calcium chloride the reactions became opaque and gelatinous. UV-vis studies revealed that calcium chloride could be exchanged for lithium chloride, delivering a heterogeneous, free-flowing reaction mixture and positively impacting the absorptivity of the EDA Scheme 2. Scale-up of EDA complex catalyzed complex. Applying this knowledge to scale up efforts, methyl N-Boc pyrrole-2-carboxylate was effectively trifluoromethylated on a $2 \mathrm{mmol}$ scale in a plug-flow reactor delivering $1.19 \mathrm{~g}(46 \%)$ of the desired product, notably, this is a 10 -fold increase in scale relative to the batch reactions (Scheme 2). trifluoromethylation in flow.

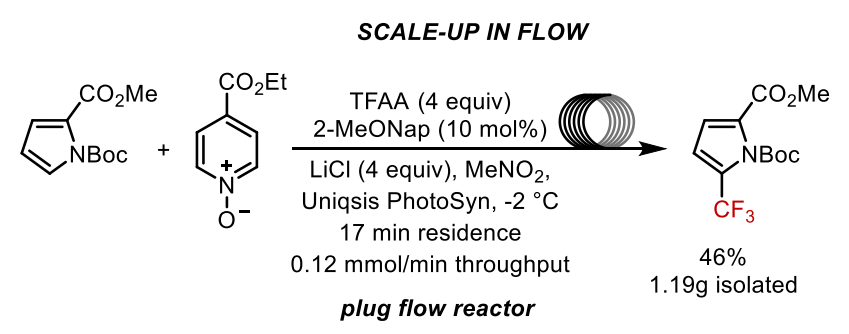

EDA complexes have been extensively studied for their ability to promote radical perfluoroalkylation reactions (14). However, these reaction manifolds are predicated on the ability of n-donors to favorably interact with the polarized C-I bond of perfluoroalkyl iodides for complexation. However, this approach prevents unified applicability across perfluoroalkylation and alkylation reaction manifolds, as less polarized alkyl iodides do not form EDA complexes. Under our EDA complex catalysis conditions, in situ generation of ethyl $N$ (pivaloyloxy)pyridinium-4-carboxylate in the presence of 2-methoxynaphthalene delivered the desired EDA complex (IV) that displayed a broad absorbance from 380-495 nm assigned as the CT bond. Subjecting this 
species to the reaction conditions in the presence of 1 equivalent of quinoxaline furnished the desired 2-(tertbutyl)quinoxaline (4a) in 57\% yield (Figure 6). Attempts to further optimize for the Minisci alkylation were unsuccessful, as alkylation ethyl isonicotinate derived acceptor was competitive with that of quinoxaline. Extension of the EDA complex catalyzed Minisci alkylation allowed for the alkylation of more complex heterocycles. Subjecting 3chloro-6-phenyl pyridazine to the reaction conditions provided tert-butylated product $\mathbf{4 b}$ in $24 \%$ yield. The imidazopyridine core structure was tert-butylated to provide structure $4 \mathbf{c}$ in $24 \%$ yield. A brief exploration of diverse radical fragments revealed that cyclohexyl, cyclopropyl, and benzyl radical fragments were efficiently generated and provided synthetically useful yields of the alkylated quinoxaline products (4d-f).

We have developed a photochemical protocol that leverages an EDA complex as a catalytic intermediate to promote the $\mathrm{C}-\mathrm{H}$ trifluoromethylation and alkylation of (hetero)arenes. The catalytic reaction is enabled by the selective donor-acceptor complexation between ethyl $\mathrm{N}$ (acyloxy)pyridinium-4-carboxylate salts and an exogenous
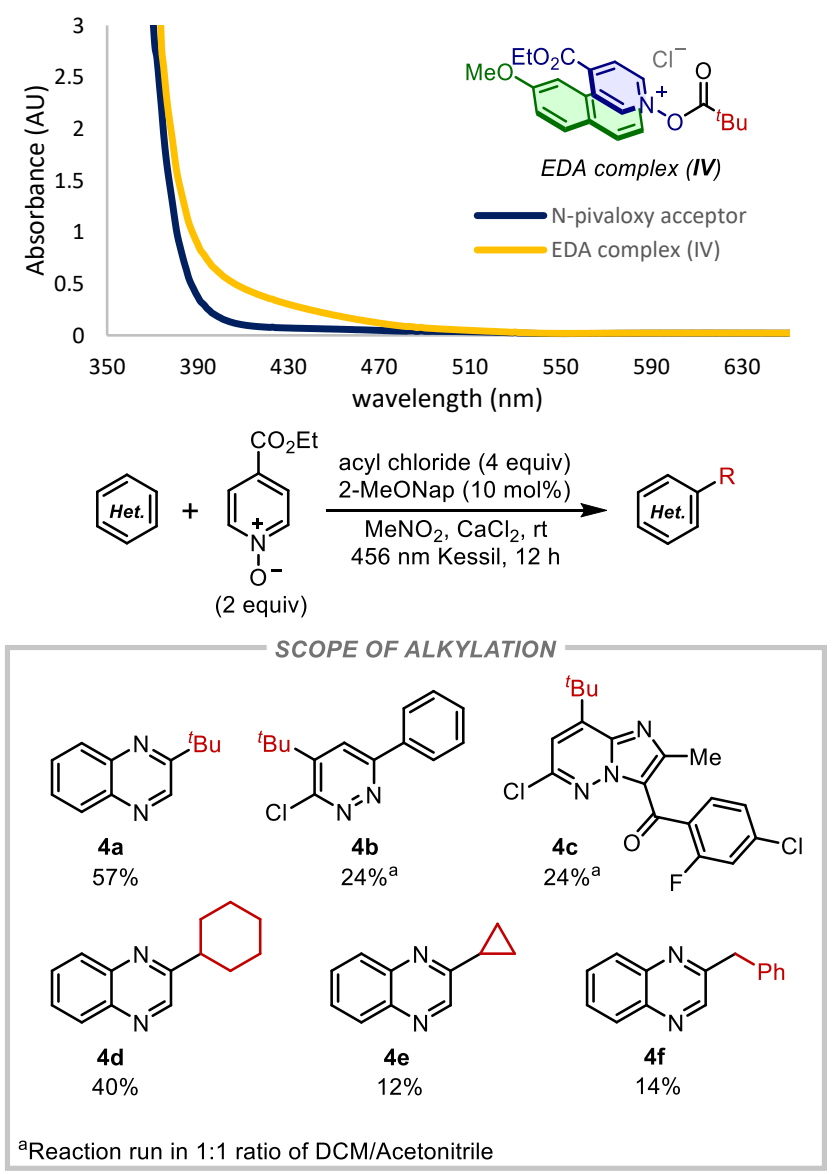

Figure 6. UV-vis of $N$-(pivaloxy)pyridinium EDA complex (top). Scope of EDA complex catalyzed Minisci alkylation (bottom).

donor, 2-methoxynaphthalene, which undergoes photochemical degradation to reveal the reactive radical intermediates. Study of the EDA complex revealed that the addition of inorganic salts, calcium chloride and lithium chloride, enhanced the absorptivity of the complex. This information allowed for the EDA complex promoted trifluoromethylation of methyl N-Boc pyrrole-2-carobylate to be carried out on multigram scale in flow.

Acknowledgment: Financial support was provided by National Science Foundation (CHE-1900266) and the University of Michigan.

\section{References}

(1) Turro, N. J.; Ramamurthy, V.; Scaiano, J. C., Supramolecular Organic Photochemistry: The Control of Organic Photochemistry and Photophysics through Intermolecular Interactions. Modern Molecular Photochemistry of Organic Molecules. University Science Books: 2010.

(2) (a) Hilinski, E. F.; Masnovi, J. M.; Amatore, C.; Kochi, J. K.; Rentzepis, P. M., Charge-transfer excitation of electron donor-acceptor complexes. Direct observation of ion pairs by time-resolved (picosecond) spectroscopy. Journal of the American Chemical Society 1983, 105 (19), 6167-6168. (b) Lee, K. Y.; Kochi, 
J. K., Charge-transfer structures of aromatic EDA complexes with N-heteroatom-substituted pyridinium cations. Journal of the Chemical Society, Perkin Transactions 2 1992, (7), 1011-1017.

(3) For a recent perspective on the synthetic applications of EDA complexes see: (a) Crisenza, G. E. M.; Mazzarella, D.; Melchiorre, P., Synthetic Methods Driven by the Photoactivity of Electron DonorAcceptor Complexes. Journal of the American Chemical Society 2020, 142 (12), 5461-5476. For selected recent examples of synthetic applications of stoichiometric EDA complexes, see: (b) Liu, B.; Lim, C.-H.; Miyake, G. M., Visible-Light-Promoted C-S Cross-Coupling via Intermolecular Charge Transfer. Journal of the American Chemical Society 2017, 139 (39), 13616-13619. (c) Börgel, J.; Tanwar, L.; Berger, F.; Ritter, T., Late-Stage Aromatic C-H Oxygenation. Journal of the American Chemical Society 2018, 140 (47), 16026-16031. (d) Yang, M.; Cao, T.; Xu, T.; Liao, S., Visible-Light-Induced Deaminative Thioesterification of Amino Acid Derived Katritzky Salts via Electron Donor-Acceptor Complex Formation. Organic Letters 2019, 21 (21), 8673-8678. (e) Lübbesmeyer, M.; Mackay, E. G.; Raycroft, M. A. R.; Elfert, J.; Pratt, D. A.; Studer, A., Base-Promoted C-C Bond Activation Enables Radical Allylation with Homoallylic Alcohols. Journal of the American Chemical Society 2020, 142 (5), 2609-2616.

(4) (a) Bockman, T. M.; Lee, K. Y.; Kochi, J. K., Time-resolved spectroscopy and charge-transfer photochemistry of aromatic EDA complexes with X-pyridinium cations. Journal of the Chemical Society, Perkin Transactions 2 1992, (9), 1581-1594. (b) Kim, E. K.; Bockman, T. M.; Kochi, J. K., Electrontransfer mechanism for aromatic nitration via the photoactivation of EDA (electron donor-acceptor) complexes. Direct relationship to electrophilic aromatic substitution. Journal of the American Chemical Society 1993, 115 (8), 3091-3104.

(5) Arceo, E.; Jurberg, I. D.; Álvarez-Fernández, A.; Melchiorre, P., Photochemical activity of a key donoracceptor complex can drive stereoselective catalytic $\alpha$-alkylation of aldehydes. Nature Chemistry 2013, 5 (9), 750-756.

(6) (a) Woźniak, Ł.; Murphy, J. J.; Melchiorre, P., Photo-organocatalytic Enantioselective Perfluoroalkylation of $\beta$-Ketoesters. Journal of the American Chemical Society 2015, 137 (17), 5678-5681. (b) Bahamonde, A.; Melchiorre, P., Mechanism of the Stereoselective $\alpha$-Alkylation of Aldehydes Driven by the Photochemical Activity of Enamines. Journal of the American Chemical Society 2016, 138 (25), 80198030. (c) Cao, Z.-Y.; Ghosh, T.; Melchiorre, P., Enantioselective radical conjugate additions driven by a photoactive intramolecular iminium-ion-based EDA complex. Nature Communications 2018, 9 (1), 3274. (d) Morack, T.; Mück-Lichtenfeld, C.; Gilmour, R., Bioinspired Radical Stetter Reaction: Radical Umpolung Enabled by Ion-Pair Photocatalysis. Angewandte Chemie International Edition 2019, 58 (4), 1208-1212.

(7) Fu, M.-C.; Shang, R.; Zhao, B.; Wang, B.; Fu, Y., Photocatalytic decarboxylative alkylations mediated by triphenylphosphine and sodium iodide. Science 2019, 363 (6434), 1429. 
(8) Bosque, I.; Bach, T., 3-Acetoxyquinuclidine as Catalyst in Electron Donor-Acceptor Complex-Mediated Reactions Triggered by Visible Light. ACS Catalysis 2019, 9 (10), 9103-9109.

(9) (a) Beatty, J. W.; Douglas, J. J.; Cole, K. P.; Stephenson, C. R. J., A scalable and operationally simple radical trifluoromethylation. Nature Communications 2015, 6 (1), 7919. (b) Beatty, Joel W.; Douglas, James J.; Miller, R.; McAtee, Rory C.; Cole, Kevin P.; Stephenson, Corey R. J., Photochemical Perfluoroalkylation with Pyridine N-Oxides: Mechanistic Insights and Performance on a Kilogram Scale. Chem 2016, 1 (3), 456-472.

(a) Nappi, M.; Bergonzini, G.; Melchiorre, P., Metal-Free Photochemical Aromatic Perfluoroalkylation of $\alpha$-Cyano Arylacetates. Angewandte Chemie International Edition 2014, 53 (19), 4921-4925. (b) Wang, Y.; Wang, J.; Li, G.-X.; He, G.; Chen, G., Halogen-Bond-Promoted Photoactivation of Perfluoroalkyl Iodides: A Photochemical Protocol for Perfluoroalkylation Reactions. Organic Letters 2017, 19 (6), 1442-1445. (c) Li, Y.; Rao, M.; Fan, Z.; Nian, B.; Yuan, Y.; Cheng, J., A visible-lightirradiated electron donor-acceptor complex-promoted radical reaction system for the $\mathrm{CH}$ perfluoroalkylation of quinolin-4-ols. Tetrahedron Letters 2019, 60 (38), 151046. (d) Liu, Y.; Chen, X.L.; Sun, K.; Li, X.-Y.; Zeng, F.-L.; Liu, X.-C.; Qu, L.-B.; Zhao, Y.-F.; Yu, B., Visible-Light Induced Radical Perfluoroalkylation/Cyclization Strategy To Access 2-Perfluoroalkylbenzothiazoles/Benzoselenazoles by EDA Complex. Organic Letters 2019, 21 (11), 4019-4024. (e) Guo, Q.; Wang, M.; Liu, H.; Wang, R.; Xu, Z., Visible-Light-Promoted Dearomative Fluoroalkylation of $\beta$-Naphthols through Intermolecular Charge Transfer. Angewandte Chemie International Edition 2018, 57 (17), 4747-4751.

(11) (a) Fukuzumi, S.; Wong, C. L.; Kochi, J. K., Unified view of Marcus electron transfer and Mulliken charge transfer theories in organometallic chemistry. Steric effects in alkylmetals as quantitative probes for outer-sphere and inner-sphere mechanisms. Journal of the American Chemical Society 1980, 102 (9), 2928-2939. (b) Nagakura, S., Mulliken's Charge-Transfer Theory and its Application to Chemical Reactions. Molecular Crystals and Liquid Crystals 1985, 126 (1), 9-18.

(12) Renny, J. S.; Tomasevich, L. L.; Tallmadge, E. H.; Collum, D. B., Method of Continuous Variations: Applications of Job Plots to the Study of Molecular Associations in Organometallic Chemistry. Angewandte Chemie International Edition 2013, 52 (46), 11998-12013.

(13) Nizhnik, Y. P.; Lu, J.; Rosokha, S. V.; Kochi, J. K., Lewis acid effects on donor-acceptor associations and redox reactions: ternary complexes of heteroaromatic $\mathrm{N}$-oxides with boron trifluoride and organic donors. New Journal of Chemistry 2009, 33 (11), 2317-2325.

(14) (a) For a review of EDA complex promoted perfluoroalkylation see: Postigo, A., Electron DonorAcceptor Complexes in Perfluoroalkylation Reactions. European Journal of Organic Chemistry 2018, 2018 (46), 6391-6404. 